\title{
The Stability of Cosmic Fullerenes and Fullerenic Aggregates
}

\author{
E. R. Micelotta ${ }^{1}$, J. Cami ${ }^{1 *}$, E. Peeters ${ }^{1 *}$, H. Zettergren ${ }^{2}$, H. T. \\ Schmidt $^{2}$, H. Cederquist ${ }^{2}$, A. P. Jones ${ }^{3}$ and A. G. G. M. Tielens ${ }^{4}$ \\ ${ }^{1}$ Department of Physics and Astronomy, University of Western Ontario, London, ON, Canada \\ email: emicelot@uwo.ca \\ *also at SETI Institute, 189 Bernardo Avenue, Mountain View, CA, USA \\ ${ }^{2}$ Department of Physics, Stockholm University, S-106 91 Stockholm, Sweden \\ ${ }^{3}$ Institut d'Astrophysique Spatiale, CNRS/Université Paris Sud, F-91405, Orsay, France \\ ${ }^{4}$ Leiden University, Leiden Observatory, NL-2300 RA Leiden, The Netherlands
}

\begin{abstract}
Establishing the stability of cosmic fullerenes and fullerenic aggregates is extremely relevant for a variety of reasons. For instance, the emission features of $\mathrm{C}_{60}$ and $\mathrm{C}_{70}$ fall in the same spectral region as the Un-identified InfraRed (UIR) bands, which they could contribute to. To be able to contribute to the UIR emission, however, fullerenes must be able to survive long enough against the destruction mechanisms operating in the interstellar medium. In this study we focus on the effects of collisional processing, i.e., the bombardment by energetic ions and electrons. A recent experimental/theoretical study has shown that ion collisions with $\mathrm{C}_{60}$ clusters result in the dissociation of the cluster with the simultaneous formation of covalent fullerene dimers, which could play a role as DIBs carriers. We present here our first results about the collisional processing of $\mathrm{C}_{60}$ molecules and clusters by $\mathrm{H}, \mathrm{He}$ and $\mathrm{C}$ ions in interstellar shocks. We have adapted the models that have previously been developed to successfully treat the collisional processing of PAHs in space. The nature of the interaction and the similarities between PAHs and fullerenes make this approach appropriate. In addition, our study shows that the formation of covalent dimers following ion collisions with $\mathrm{C}_{60}$ clusters is compatible with the astrophysical conditions under consideration.
\end{abstract}

Keywords. ISM: molecules, molecular processes, shock waves.

\section{Introduction}

Fullerene molecules $\mathrm{C}_{60}$ and $\mathrm{C}_{70}$ (Cami et al. 2010; Cami 2012, and references therein) and possibly $\mathrm{C}_{60}$ clusters (Evans et al. 2012) have recently been detected in extraterrestrial environments. These species may be connected with the carriers of the Diffuse Interstellar Bands (DIBs). It is of particular interest to establish whether fullerenes and fullerenic aggregates can survive the bombardment by energetic ions and electrons, usually identified as "collisional processing". This processing is very common in the interstellar medium and it is due to high velocity projectiles arising from interstellar shocks, hot gas and cosmic rays. In previous studies (Micelotta et al. 2010a,b, 2011) it has been demonstrated that interstellar Polycyclic Aromatic Hydrocarbons (PAHs) are easily destroyed by collisional processing, revealing that these species are much less resistant than previously thought. The investigation of the stability of fullerenes in space is therefore a natural extension of our previous work.

Zettergren et al. (2013) have recently shown that collisions between $22.5 \mathrm{keV} \mathrm{He}^{2+}$ ions and van der Waals clusters of $\mathrm{C}_{60}$-fullerenes result not only in the ionisation and dissociation of the cluster but also in the formation of covalently bound dumb-bell fullerene 
dimers. He ions with comparable energies exist in space. Our investigation will allow to determine whether such dissociation/formation reactions can occur over the whole range of projectiles, energies and physical conditions which characterize collisional processing in extraterrestrial environments.

\section{The stability of $\mathbf{C}_{60}$ molecules and clusters in the laboratory}

Collisions between $22.5 \mathrm{keV} \mathrm{He} \mathrm{H}^{2+}$ ions and van der Waals clusters of $\mathrm{C}_{60}$-fullerenes have recently been investigated by Zettergren et al. (2013) from an experimental and theoretical point of view. The collision products have been measured by a linear timeof-flight mass spectrometer. Surprisingly, the dominant molecular fusion products are dumb-bell systems $\mathrm{C}_{119}^{+}$and $\mathrm{C}_{118}^{+}$. For a detailed discussion about the findings, we refer the reader to the original paper from Zettergren et al. (2013). For our present purposes we would like to highlight the following.

Fig. 1 shows the measured mass-to-charge distributions for ions detected in coincidence with intact $\mathrm{C}_{60}^{+}$ions. The dominant intensity of the $\mathrm{C}_{60}^{+}$peak clearly indicates that the fragmentation of individual ions is a very rare event in collisions between $\mathrm{He}^{2+}$ ions and van der Waals clusters of $\mathrm{C}_{60}$ molecules. In addition, in the region of high mass-tocharge ratios (right panel in Fig. 1) the spectrum is dominated by the three peaks at the positions for 120,119 and $118 n_{\mathrm{C}} / e$. The peak at $n_{\mathrm{C}} / e=120$ may be due to $\left[\mathrm{C}_{60}\right]_{2}^{+}$dimers remaining after the dissociation of larger clusters and/or to the formation of covalently bound systems through the reaction $\mathrm{C}_{60}^{+}+\mathrm{C}_{60} \rightarrow \mathrm{C}_{120}^{+}$. In contrast, the peaks at $n_{\mathrm{C}} / e=$ 119 and 118 are most likely due to the formation of covalently bound dumb-bell systems through the low energy reactions $\mathrm{C}_{59}^{+}+\mathrm{C}_{60} \rightarrow \mathrm{C}_{119}^{+}$and $\mathrm{C}_{58}^{+}+\mathrm{C}_{60} \rightarrow \mathrm{C}_{118}^{+}$.

The key process here is the impact of the $\mathrm{He}^{2+}$ ion. Among the ion trajectories penetrating the cluster, those sufficiently close to an individual carbon atom will be able to knock it out, producing $\mathrm{C}_{59}^{+}$inside the cluster. This prompt knock-out process is known as nuclear interaction. $\mathrm{C}_{59}^{+}$is very fragile, thus a second carbon atom may be easily lost, producing $\mathrm{C}_{58}^{+}$. Both these ions are much more reactive (with $\mathrm{C}_{60}$ ) than $\mathrm{C}_{60}^{+}$.

The formation mechanism of the dimers has been elucidated through Molecular Dynamics (MD) simulations by Zettergren et al. (2013) and the results are shown in their Fig. 3. The simulation starts immediately after the prompt (femtosecond) ejection of a single carbon atom from a single $\mathrm{C}_{60}$ molecule - here the central molecule in a $\left[\mathrm{C}_{60}\right]_{13}$ cluster with the archetype icosahedral geometry. Within a few picoseconds the $\mathrm{C}_{59}^{+}$ion, formed via nuclear interaction with the impacting $\mathrm{He}^{2+}$, collides with a neighbour $\mathrm{C}_{60}$ molecule forming a $\mathrm{C}_{119}^{+}$system. Calculations by Zettergren et al. (2013) have shown that the adiabatic dissociation energies for $\mathrm{C}_{119}^{+}$and $\mathrm{C}_{118}^{+}$are higher than the one for $\mathrm{C}_{120}^{+}$ $(5.4 \mathrm{eV}$ and $3.0 \mathrm{eV}$ against $1.0 \mathrm{eV}$ ). Therefore, the former are expected to survive longer after their formation. Stopping calculations have shown that a significant fraction of the energy carried by the $\mathrm{He}^{2+}$ projectile is transferred to the electron clouds of the whole target cluster (electronic interaction), leading to the dissociation of the cluster, but very rarely to the fragmentation of single molecules. This is consistent with the experimental findings (cf. Fig. 1). Through the rapid sharing of the excitation energy on the whole cluster, individual molecules such as the fragile $\mathrm{C}_{59}^{+}$can then become sufficiently cold to stay intact and react with another $\mathrm{C}_{60}$ inside the cluster before further decays occur.

This study shows that collisions between $22.5 \mathrm{keV} \mathrm{He}^{2+}$ ions and van der Waals clusters of $\mathrm{C}_{60}$ molecules results in two phenomena: $\left.\mathrm{i}\right)$ the ionisation and dissociation of the cluster and ii) the formation of covalent $\mathrm{C}_{118}^{+}$and $\mathrm{C}_{119}^{+}$dumb-bell dimers inside the fragmenting cluster. These processes have profound astrophysical implications which are discussed in the following section. 


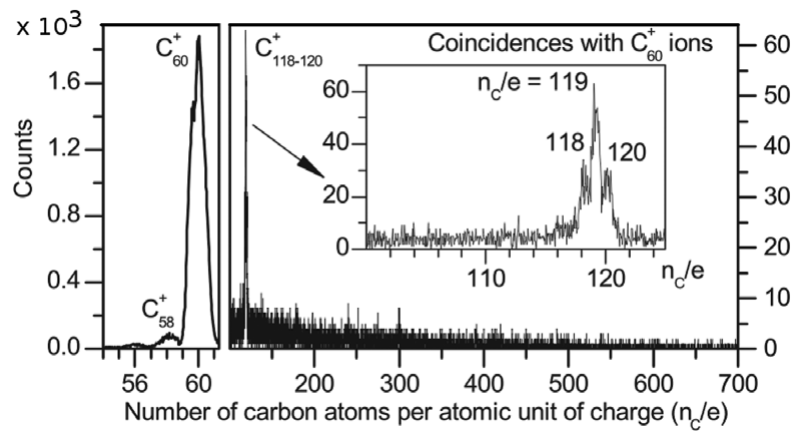

Figure 1. The mass-to-charge distribution measured after $22.5 \mathrm{keV} \mathrm{He}{ }^{2+}+\left[\mathrm{C}_{60}\right]_{\mathrm{n}}$ collisions, for events detected in coincidence with intact $\mathrm{C}_{60}^{+}$ions. Figure reproduced with permission from Zettergren et al. (2013, PRL, 110, 185501). Copyright (2013) by the American Physical Society.

\section{The stability of $\mathbf{C}_{60}$ molecules and clusters in space}

The first, fundamental step for the dissociation/formation reaction in $\mathrm{C}_{60}$ clusters is the prompt ejection of a single carbon atom from one $\mathrm{C}_{60}$ molecule within the cluster, following collisions with ions. We need to establish whether this process occurs under interstellar conditions. For this study we focus on collisional processing in interstellar shocks with velocities between 50 and $200 \mathrm{~km} \mathrm{~s}^{-1}$ (Micelotta et al. 2010a), where the considered projectiles, $\mathrm{H}^{+}, \mathrm{He}^{+}$and $\mathrm{C}^{+}$, have energies between $\sim 10 \mathrm{eV}$ and $\sim 10 \mathrm{keV}$.

The knock-out process is due to the so-called nuclear interaction, where a certain amount of energy is transferred during a binary collision between the incoming projectile and one single atom (carbon in our case) in the $\mathrm{C}_{60}$ target. To describe the nuclear interaction of $\mathrm{C}_{60}$ we have adapted the formalism developed by Micelotta et al. (2010a) for PAH targets. We refer the reader to this paper for the explanation of the model. For the sake of clarity, we report here the essential concepts and the equations (without derivation) which have been used for this work.

If the energy transferred during the binary collision exceeds a specific threshold, $T_{0}$, the recoiling atom is ejected. The nuclear threshold energy depends on the target material. For $\mathrm{C}_{60}$ we adopt the value $T_{0}=15 \mathrm{eV}$, consistent with experimental determinations by Füller \& Banhart (1996) and recent theoretical studies by Zettergren et al. (2013). For comparison, the equivalent value that was assumed for PAHs is $T_{0}=7.5 \mathrm{eV}$ (Micelotta et al. 2010a).

We are interested in destructive collisions only, i.e., collisions where the transferred energy exceeds the threshold $T_{0}$. This case was not treated in previous models (e.g., Sigmund 1981), which only considered the specific situation where $T_{0}=0$. To include the treatment of collisions above threshold $\left(T_{0}>0\right)$ we modified the existing theory as described in Micelotta et al. (2010a), obtaining the following expressions for the quantities relevant to this work. For the nuclear stopping cross section of a particle with energy $E$ we have

$$
S_{\mathrm{n}}(E)=\int_{T_{0}}^{T_{\mathrm{m}}} \mathrm{d} \sigma(E, T) \cdot T=S_{\mathrm{n}}^{0}(E)\left[1-\left(\frac{E_{0 \mathrm{n}}}{E}\right)^{1-m}\right]
$$

where $S_{\mathrm{n}}^{0}(E)$ is the nuclear stopping cross section when no threshold is considered $\left(T_{0}=0\right)$, $T$ is the amount of energy transferred to the target and $T_{\mathrm{m}}$ is the maximum transferable energy, corresponding to a head-on collision. The quantity $E_{0 n}$ represents the energy required for the projectile to transfer the energy $T_{0}$ in a head-on collision. For the 

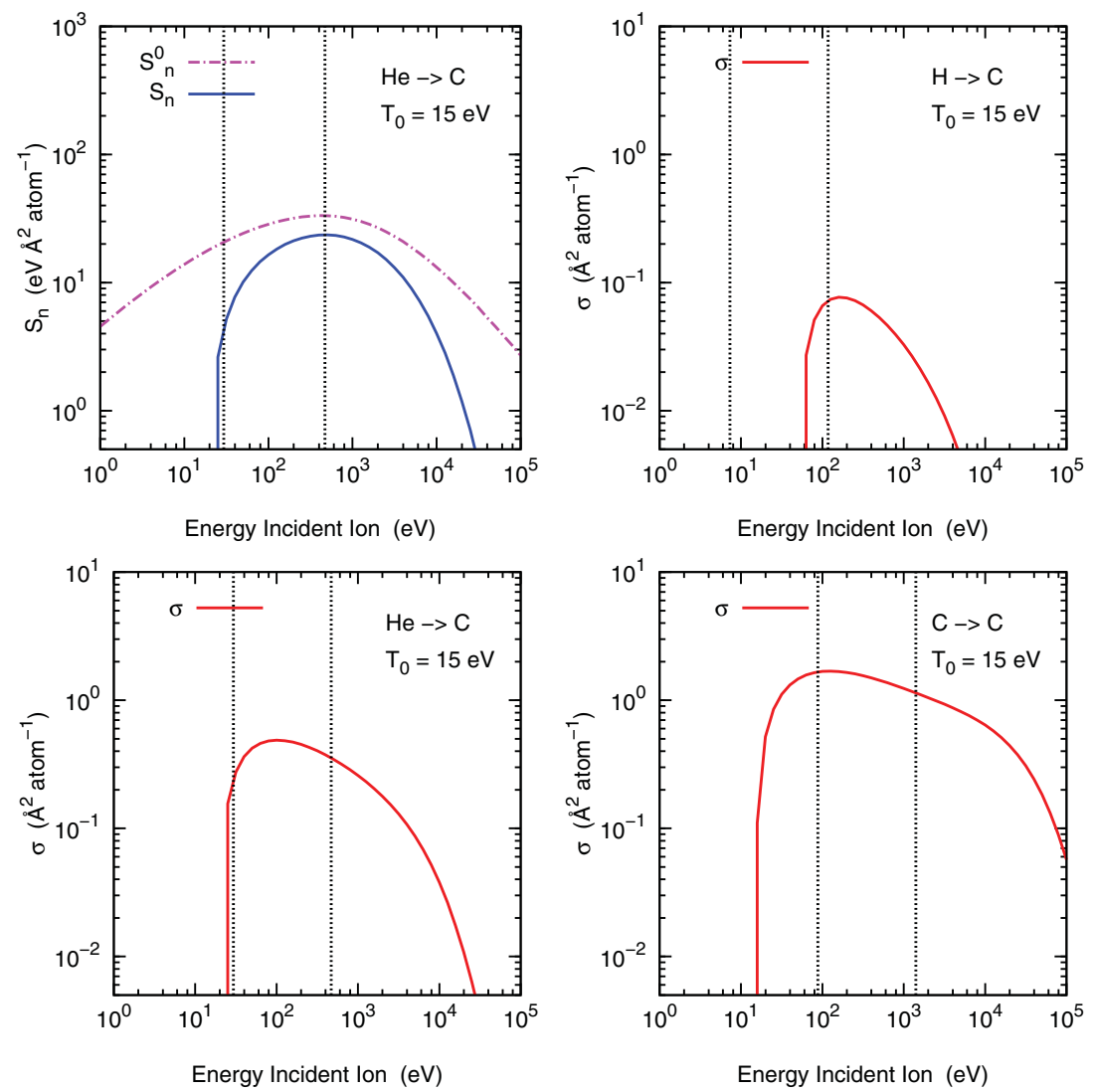

Figure 2. The nuclear stopping cross section $S_{\mathrm{n}}(E)$ for $\mathrm{He}^{+}$projectiles and the total cross section $\sigma(E)$ calculated for $\mathrm{H}, \mathrm{He}$ and $\mathrm{C}$ ions impacting on a single carbon atom in a $\mathrm{C}_{60}$ molecule. The two vertical lines indicate the minimum and maximum energies for the incident ion. These are defined as the kinetic energies of the projectile when its velocity $v_{\mathrm{p}}$ equals $\frac{3}{4}$ $\left(v_{\mathrm{S} 1, \mathrm{~S} 2}\right)$, where $v_{\mathrm{S} 1}=50 \mathrm{~km} \mathrm{~s}^{-1}$ and $v_{\mathrm{S} 2}=200 \mathrm{~km} \mathrm{~s}^{-1}$ are the lowest and highest shock velocities considered in this study.

differential cross section, $\mathrm{d} \sigma(E, T)$, we adopt an approximated expression calculated using the power approximation of the Thomas-Fermi model of interatomic interaction, i.e. with a potential in the form $V(r) \propto r^{-1 / m}$, where $r$ is the distance between colliding nuclei and $m$ is a parameter related to the steepness of the interatomic potential. The quantity $m$ can be interpreted as an indicator of the energy of the projectile, varying slowly from $m=1$ at high energies to $m \approx 0$ at low energies.

The total energy transfer cross section per carbon atom, $\sigma(E, T)$, is given by

$$
\sigma(E)=\int_{T_{0}}^{T_{\mathrm{m}}} \mathrm{d} \sigma(E, T)=S_{\mathrm{n}}^{0}(E) \frac{1-m}{m} \frac{1}{\gamma E}\left[\left(\frac{E_{0 \mathrm{n}}}{E}\right)^{-m}-1\right] ; \gamma=\frac{4 M_{1} M_{2}}{\left(M_{1}+M_{2}\right)^{2}}
$$

where $M_{1}$ and $M_{2}$ are the mass of the incident and target particle respectively.

Fig. 2 shows $\sigma(E)$ calculated for $\mathrm{H}^{+}, \mathrm{He}^{+}$and $\mathrm{C}^{+}$colliding with $\mathrm{C}_{60}$ together with $S_{\mathrm{n}}^{0}(E)$ and $S_{\mathrm{n}}(E)$ for $\mathrm{He}^{+}$projectiles as an example. (Micelotta et al. 2014). It can be seen that the threshold $T_{0}=15 \mathrm{eV}$ has a dramatic effect on the nuclear stopping cross section. The characteristic convex shape for both $S_{\mathrm{n}}$ and $\sigma$ clearly illustrates that nuclear energy transfer is important only when the energy of the projectiles falls into a specific 
range. Projectiles in interstellar shocks have energies in the window delimited by the two vertical lines in Fig. 2. This figure shows that, in case of $\mathrm{H}$, only the ions in the highest velocity shocks are energetic enough to cause carbon ejection. In contrast, $\mathrm{He}$ and $\mathrm{C}$ have energies corresponding to high values of $\sigma$. Their contribution to nuclear stopping, hence carbon ejection, is large for all the shock velocities considered here. However, we will have to consider the effective abundance of each ion. Because of the low abundance of carbon with respect to helium $\left(\chi_{\mathrm{C}}: \chi_{\mathrm{He}}=10^{-4}: 10^{-1}\right)$, we expect the contribution from carbon to be heavily reduced. On the other hand, He has a relatively high abundance coupled with a strong contribution to nuclear stopping. Thus, He is potentially the major agent for prompt ejection of carbon atoms from $\mathrm{C}_{60}$ molecules in interstellar shocks.

\section{Conclusions and perspectives}

With our previous work (Micelotta et al. 2010a), this study provides a set of analytical expressions to describe the nuclear stopping above threshold for large molecules and small clusters under astrophysical conditions. Because of their size, these entities represent an intermediate population between 'classical' small molecules and solids. Therefore, nuclear stopping cannot be treated as regular molecular dissociation nor in terms of sputtering of bulk materials. Our model provides the appropriate formalism to approach this problem.

Our calculations show that the type of projectiles and the energy conditions in interstellar shocks are compatible with the prompt ejection of a single carbon atom from $\mathrm{C}_{60}$ molecules. This is the first, fundamental step for the formation of covalent fullerene dimers along the pathway identified by Zettergren et al. (2013). The following step is to convolve our results over the actual velocity, density and temperature profiles of the projectiles along the shock. This will provide the total timescale of the process, thus a measure of the effective stability of cosmic fullerenes and clusters of fullerenes against nuclear interaction in space. The stability of such entities versus collisional processing will be completed studying the effect of electronic stopping and of collisions with electrons. The natural follow-up of these studies will be to establish the stability of the newlyformed dimers, together with their emission characteristics. This will allow to determine: i) whether or not we could expect to find them in space and ii) how we could identify them in astronomical spectra.

This work and the proposed follow-ups are part of a larger project aiming to investigate the collisional processing of cosmic macromolecules and clusters. This requires taking into account for the first time the very specific (and largely unexplored) physical and chemical properties deriving from their nanometric scale.

\section{References}

Cami, J. 2012, Proc. IAU General Assembly, Beijing, Aug 27-28 , 2012, arXiv:1210.1730

Cami, J., Bernard-Salas, J., Peeters, E., \& Malek, S. E. 2010, Science, 329, 1180

Evans, A., van Loon, J. T., Woodward, C. E., et al. 2012, MNRAS, 421, L92

Füller, T. \& Banhart, F. 1996, Chem. Phys. Lett., 254, 372

Micelotta, E. R., Cami, J., Peeters, E., et al. 2014, in preparation

Micelotta, E. R., Jones, A. P., \& Tielens, A. G. G. M. 2010a, A\&A, 510, A36

-. 2010b, A\&A, 510, A37

—. 2011, A\&A A, 526, A52

Sigmund, P. 1981, Sputtering by Particle Bombardment Vol.1, ed. R. Behrisch, in Topics in Applied Physics, Vol. 47 (Springer - Verlag)

Zettergren, H., Rousseau, P., Wang, Y., et al. 2013, Phys. Rev. Lett., 110, 185501 